\title{
Transformations, Opportunities and Challenges: How Government Copes with Online Public Opinion in Big Data Era
}

\author{
Jingyu Chen \\ School of Languages and Media, Anhui University of Finance and Economics, Bengbu, China \\ Email: mlbsabc@sina.com
}

How to cite this paper: Chen, J.Y. (2019) Transformations, Opportunities and Challenges: How Government Copes with Online Public Opinion in Big Data Era. Open Journal of Social Sciences, 7, 424-437. https://doi.org/10.4236/jss.2019.74034

Received: April 4, 2019

Accepted: April 26, 2019

Published: April 29, 2019

Copyright $\odot 2019$ by author(s) and Scientific Research Publishing Inc. This work is licensed under the Creative Commons Attribution International License (CC BY 4.0).

http://creativecommons.org/licenses/by/4.0/

\section{c) (i) Open Access}

\begin{abstract}
With the popularization of portable terminal equipment and cyber social contact media, there are fewer and fewer barriers against expression of public opinion, which has led to tremendous structural changes in public opinion field. Online public opinion monitoring in government has, therefore, undergone a dramatic transformation in working methods, managerial concepts and data analysis technologies in order to keep pace with the times. For this purpose, the paper tries to offer a new insight into transformation and challenges of online public opinion in the big data era and puts forward some countermeasures as follows: 1) Construct a government platform for online public opinion to improve information collection. 2) Employ an open data policy to enhance the government's public credibility. 3) Cultivate more professionals and maintain a sustainable development in research and development as well as technology innovation. 4) Improve government officials' media literacy and strengthen their awareness of online public relations. 5) Build various types of case data analysis database to extract and purify the will of people, to gather in one place the wisdom of people and to construct a think tank of all the people for decision making.
\end{abstract}

\section{Keywords}

Big Data, Government Monitoring, Transformations, Challenges, Countermeasures

\section{Introduction}

Viktor Mayer-Schönberger once said, "The essence of the world is data, and big data will usher in a significant era of transformation" [1]. As a matter of fact, 
human beings have undergone a 5000-year-long agriculture civilization (Agricultural Revolution), a 300-year-long industrial civilization (Industrial Revolution) and are now confronted with a brand new digital civilization (IT Revolution), all of which have brought about a huge and profound influence in their own times. However, if a person wants to get a clear understanding of development tendency in industry, he or she has to see clearly what era the industry is in. Big data development is closely linked to the Internet and the derivative mass data from it has rushed the world into a big data era. Alvin Toffler (1980) first created the concept of Big Data and then data mining technology followed in 1989. It was not until 2004 that social contact media appeared and every netizen owns a private network information system, through which the net users can make and generate data continuously. In 2009, microblog sprang up and then WeChat etc. came into everyone's daily life. As a result, big data, cloud storage, cloud computing and algorithm have deeply penetrated into every social field and consequently the biggest datum explosion of all in history followed. Till 2013, the first year of big data, the data volume has exceeded eight hundred million TB, twice as the size as that in 2012. It is estimated that the data volume will surpass 16 thousand million TB in 2020, twenty times as the size as that in 2013 [2]. In 2016, the technical bottleneck that has constrained big data was entirely broken and naturally big data has become an important tool in all industries. $\mathrm{Xu}$ Changliang, chief inspector in big data business division Ali Cloud, believed that 2016 was the first year of trillion data industry and was also called the first year of mobile live streaming broadcast [3]. With the development of information technology, big data has become the most prominent symbol in the world. But as a matter of fact, the big of big data is just a relative concept and it mainly has four characters [4]: 1) Huge information volume storage capacity. TB has been upgraded into EB; 2) Rich and diversified varieties. Data can be expressed in any forms of texts, pictures, weblogs, voice files, video media and so on; 3) Great value but with a loose density. Although big data is of great value, the amount of online information is massive, chaotic and fragmented, so it needs realigning to disregard the useless and extract the essence; 4) High velocity. China mobile cyber communication was already in the stage of 4G+ in 2017 and its downward peak rate has amounted to $500 \mathrm{Mbps}$ or over. At the meantime, 5G technology has been around the corner and mobile cyber communication velocity will be once again accelerated greatly. China's " $13^{\text {th }}$ Five-Year Plan" points out that China will go all out to promote 5G and 5G commercial to be launched in 2020 will play a comprehensive role in the whole Plan. On March 30, 2019, China Mobile Group and Shanghai Municipal Government jointly announced that the world's first "double gigabit area" has been set up in Shanghai's Hongkou District and the first live 5G mobile phone call has been made at the same time, indicating that Shanghai has become China's first mobile 5G trial commercial city. By 2021, Shanghai will have achieved all-around coverage of 5G networks. Besides, consumers in more than 20 first- and second-tier cities across China, including Beijing, Shanghai, Guangzhou, Shenzhen, Tianjin, Qingdao, Suzhou and 
Shenyang, will take the lead in experiencing the convenience brought by 5G. By 2020, most provincial capitals and some second- and third-tier cities on China will also have achieved 5G coverage [5], which indicates that 2019 will be a transitional period for China from $4 \mathrm{G}+$ to $5 \mathrm{G}$. There is no doubt that the rapid development of the Internet will further promote the changes of a big data era and government governance.

\section{Development of Internet Technology Will Promote Development of Government Management}

The connection between science and technology and politics is ubiquitous. The development and application of core technology in big data will have a significant influence on the operation mode of social economy and industry and political expression and participation, which therefore leads to profound changes in government management.

In the times of Web1.0 (Internet used to mean websites and computers), say in 1990s, the establishment of large-scale portal websites for public communication was only sponsored by government agencies and almost all the chief positions were dominated by government. All the websites were government-centered and hence the government played a dominant and unparalleled role in public affairs. In addition, government determined all the services offered by the websites so duplicate efficiency, low effectiveness and one-dimension phenomenon often occurred. In addition the volume of personal website visits was, comparatively speaking, laggard.

Not until 2004 did online social contact era based on Web2.0 come into being (mobile Internet used to mean social contact media and intelligent terminals). The websites were no longer government-centered and took on more personalized styles. The websites have changed dramatically from the original PC desktop system to individual-oriented mass communication on the basis of social platforms and mobile terminals. UGCs from Blog, Tag, SNS, RSS, wiki, WeChat, etc. proved that personalized media and communication had been intensified and we-media era was approaching. In the era of government web2.0, social contact media attached much importance to public-oriented policy and the government was more active in disclosing information and absorbing external communication, interaction and participation. What's more, behaviors and processes in governance are more in line with public expectations. Some government agencies have adapted to the trend by providing mobile phone apps, public WeChat, microblog accounts and other online enquiry or governance systems to let the public participate in government management. Although Web2.0 promotes interaction and information sharing in a bidirectional dimension, duplicate efficiency and low effectiveness will also occur sometimes.

Web3.0 indicates that the era of large-scale online interconnection will come true, in which comprehensive interaction, accurate perception and expression, and intelligence interpretation between people, people and things will be rea- 
lized. Human beings will get to a higher form of harmonious wisdom. In the era of government Web3.0, big data is individual-oriented, in which the government has to place more emphasis on the openness of data, pay more attention to sharing and co-operation in order to obtain a managerial mode of sharing and co-governance, such as automatic driving technology. Consequently, government monitoring will confront very significant changes and adjustments. In a word, government Web3.0 is more efficient, effective, collaborative, co-managerial, multi-dimensional and customized, in which more civil rights and multi-dimensional interaction and cooperation are needed.

From the above, it is the technology in an era that has led to three modes and characteristics of network application. If the essence of Web1.0 is a kind of reunion, the essence of Web2.0 is interaction, while Web3.0 is a revolution of Web2.0 in order to get a better data application. For China, Web3.0 is a good start of Intelligence China. Among these three modes, government Web3.0 represents a brand new governance model and thus becomes a target in government management innovation. On March $3^{\text {rd }}$, 2015 Premier Li Keqiang delivered the government work report in the third session of the 12th National People's Congress and proposed the Internet plus plan for the first time to stress the importance of developing smart cities, which indicates that China has opened the prelude to construction of intelligence era. On March 16, 2016 five innovative ideas were reviewed and approved at the fourth session of the $12^{\text {th }}$ National People's Congress. It is universally agreed that continuous innovation in theory, system, science and technology and as well culture will be in harmony with scientific outlook on development and will be a core of China's overall development [6]. On December $9^{\text {th }}$, 2017 President Xi Jinping stressed during the second collective learning session of the Political Bureau of the CPC Central Committee "Big data should be used to enhance the modernization of national governance, and it is necessary to establish and perfect the mechanism of megadata-based scientific decision making and social governance" [7].

\section{Transformations in Mindsets Will Bring New Opportunities for Online Public Opinion Governance}

The technology in the big data era will bring technical advantages to online public opinion management. The big of big data not only means the rapid growth and massive accumulation of data but also refers to a new ability in current society, namely, the ability of obtaining huge data value through mining and analyzing massive data, which has an unprecedented product and service value. With the rapid development of the Internet, big data has been constantly integrated with cloud computing, Internet of things, mobile Internet and other new information technologies. This has brought about a change in thinking of big data and thus brought new opportunities and challenges to public opinion monitoring in the government and social organizations. Let the data speak. According to the correlation and quantitative analysis of all kinds of data, make the 
analysis of online public opinion more objective and comprehensive, so as to offer a deep insight into the situation of online public opinion. Scientific analysis and decision-making based on big data is the inherent demand and inevitable choice for improving the government's ability to study, judge and coping capacity, improving social governance, maintaining social stability and healthy development, improving government service level and realizing the modernization of government management. Governments of all countries hope to improve public opinion guidance and management ability of various social undertakings through big data. In 2012, the United States invested 200 million dollars to launch the "big data development research program". In July 2012, Japan put forward the "new ICT plan", focusing on the research and application of big data. In January 2013, the UK announced a 189-million-pound investment in big data projects such as earth observation, health and energy efficiency computing [8]. China now has the largest number of Internet users in the world. According to the $42^{\text {nd }}$ statistical report on the development of Internet in China released by China Internet Network Information Center, the number of Internet users in China reached 802 million in June 2018, with an Internet penetration rate of $57.7 \%$. 29.68 million new Internet users were added in the first half of 2018, up 3.8 percent from the end of 2017. The number of mobile Internet users in China reached 788 million, and the proportion of Internet users accessing the Internet through mobile phones amounted to 98.3 percent [9]. Therefore, the $13^{\text {th }}$ Five-Year Plan period is the decisive stage to complete the building of a moderately prosperous society in all respects, the starting stage to achieve new breakthroughs in the transformation of information and communication technology, and the expansion stage to fully release digital dividends [10].

Online public opinion is "the will of netizens", which is the sum of subjectively oriented cognition, emotion, attitude and opinion of hot events and things concerned by all social groups [11]. The promotion and popularization of smart phones accelerates the spread of online public opinions. Microblog, WeChat and other social media, mobile clients, online BBS, community, news sites and video websites constantly emerge, the change of propagation modes has led to new characteristics of online public opinion expression, which puts forward a new subject for online public opinion to study and thus results in microblogging public opinions, WeChat public opinions, the mobile phone APP BBS public opinions, video public opinions and other new types of public opinions. The "three micros and one terminal" mode represented by WeChat, microblog, micro-videos and clients has become a new focus of Chinese Internet public opinion. The virtual cyber society has been created and online expression and communication has brought about the realistic challenges in social reforms. The virtual cyber space is different from the traditional media in terms of social attributes, social characteristics, social awareness, culture, value and structure. In addition, compared with traditional media, new media have shorter communication cycle and faster speed. Meanwhile, by virtue of mobile Internet, it breaks 
through the boundary coverage and expands the communication scope to the largest extent. For example, Jiangsu 12345 hotline, the first online service and big data platform with comprehensive coverage and unified linkage in the provinces, cities and counties in China, has broken through the omnimedia and integrated many multiple media ports such as phone, microblog, WeChat and client side. The platform receives feedback from the public, and supports the comprehensive connection with the government affairs service network and mated consulting services. By analyzing the massive public opinion data generated by the platform, relevant departments can timely adjust policies to promote scientific decision-making. At present, Jiangsu 12345 online service platform has collected 13.96 million public demands of the whole province, and established such the theme pool of big data as "Jiangsu Big Visit", "No.1 Answer" of government affairs service, urban governance and service, and emergency planning. Weekly reports to the Jiangsu provincial party committee and the provincial government main leaders of the province's hot-spot special report and regularly special reports have accumulated a large number of first-hand data resources, which can help find and solve social problems in a convenient, early and comprehensive way. The platform has also set standards to build a unified data exchange system across the province and promote real-time data sharing on purpose [12], which can fully depict the new governance model and the new target selection model represented by government Web3.0.

From the above, it can be seen that big data generated in the Internet era is not only a kind of resource, a new branch of science but also a corresponding data processing technology and a new way of thinking, which requires us to embrace innovation consciousness to face a new epoch with a big data way of thinking. Only in this way can we vigorously promote the prosperity of our motherland and realize the great rejuvenation of the Chinese nation.

\section{Characteristics and Challenges of Online Public Opinions in a Big Data Era}

\subsection{Characteristics of Online Public Opinions in a Big Data Era}

With the emergence and rapid popularization of online social media, attractive netizens gradually become the main body of public opinion communication. Netizens can comment on public events or government decisions at any time, forming online public opinions and forcing the government to pay attention. In this way, ordinary netizens have become creators and disseminators of online information instead of passive receivers of information. They also improve the speed and initiatives of information transmission. Online public opinion becomes freer and more difficult to control then because of its convenience, freedom, diversity, interaction and suddenness. Therefore, public opinion monitoring has transformed from a single mode to a multiple-layer mode, from information monitoring to study, judgment, persuasion and processing. At present, online public opinion monitoring should be carefully planned in the top-level 
design with a scientific management concept to help government to collect online public opinions, which will in return be an important platform for the government to make decisions.

\subsection{Challenges of Online Public Opinions in a Big Data Era}

\subsubsection{Online Public Opinion Monitoring Technology in Big Data Has a Bottleneck}

Currently, big data processing technology and related supporting software can hardly meet with a large amount of diversified data. When it comes to online public opinion monitoring, Chinese government also needs to solve a variety of technical problems. For example, the infinity of network data aggravates the scattered and chaotic state of public opinion. Openness of information, convenience of communication and decentralization of discourse power have intensified the extreme emotions of public opinions and increased the difficulty of public opinion management. Big data is mainly composed of structured data, semi-structured data and unstructured data. Multi-channel release means makes it harder to study and judge public opinion, especially the size and structure of social media data generated by microblog, WeChat, QQ pictures and videos, etc. is totally different, because there is no rigorous structure known as unstructured data and semi-structured data, even if structured, it is neither matrix nor a cassette, so the original simple sensitive word-screening analysis method is very hard to work. The processing of this part of social contact data is far more difficult than that of structured data, and it is also a difficult problem for public opinion work analysis. At present, there are many semi-structured and unstructured data resources, accounting for $75 \%$ of the world's data [13] and there is still a lack of substantial breakthrough and progress in processing unstructured massive data intelligently. Distributed processing technology has been unable to meet the current demand since the complex diverse massive data has reduced the degree of data visualization. On the other hand, cloud computing, visual computing and other technologies update at a slower speed, and they are still in the stage of being developed and tested. More powerful technology and tools such as data mining language processing are to be introduced into the government public opinion management system, of which the application of data mining technology can help users find a large number of useful information from the text through online public opinion monitoring. With the aid of forecasting, contrast, clustering, and other functions, users can find out the connection within the mined data to judge the present situation and trend of online public opinion development.

\subsubsection{Local Government Lack Professional Organizations and Personnel,} and Its Level of Data Monitoring and Analysis Is Not High Either

At present, China's domestic online public opinion monitoring institutions can be broadly classified into three types [14]: 1) Online public opinion monitoring organizations and service systems led by mainstream media, such as People's Daily online, Xinhua online and Procurators daily and others. These institutions 
can provide public opinion monitoring and analysis services for Chinese government and public administration departments; 2) Online public opinion monitoring agencies in universities, academic institutions and research centers. These institutions are generally not for commercial or profit purposes, but more for academic researches; 3) Public opinion information companies established by software companies or professional market research institutions. This type of public opinion information company not only serves the government and public management departments, but also provides a powerful public opinion monitoring support for large and medium-sized enterprises. For local governments, it is difficult to set up a professional public opinion management team due to the lack of capable organizations or able technical personnel, so they can only outsource the monitoring to a third party, a public opinion information company. Since outsourced information companies lack the authority to monitor the online information in specific social management areas, they cannot guarantee the desired analysis and monitoring results of online public opinions government departments want, not to mention new technologies and methods used to collect and process online public opinion information within government departments. Therefore, local governments attach great importance to the cultivation of public opinion monitoring analysts and construction of organizations, which is an inevitable means to improve the level of professional analysis and coping capacity. Cao Lubao, a member of the standing committee of Nanjing municipal party committee and the minister of publicity, proposed at the 2018 Nanjing Think Tank Summit on Internet Big Data and Social Governance: "Studying cyberspace from the perspective of public opinion may play a more significant role in promoting the development of smart cities and improving social governance" [15]. Therefore, in order to realize "digital China and a smart society", talents should come first.

\subsubsection{Data Security and Privacy Are Important Issues for Government Governance}

In a big data era, we must pay attention to data security and citizens' privacy protection. Otherwise, once hot events happen, some people will indiscriminately turn to cyber man-hurt and will publicly expose the privacy of the parties concerned and infringe the interests of others. On the other hand, it may be used by others or foreign hostile forces to create and spread rumors, or to seek personal gains, or to earn illegal economic interests, or even to induce criminal crimes, which may cause immeasurable losses to others or the country. At the beginning of 2018, Baidu and Today's Top Story Online were caught up in the hacking scandal [16], and were interviewed by relevant departments respectively. Later on, the two companies made quick clarifications. Now a lot of social contact software packages have the problem of privacy disclosure, of which having access to users' contact lists has become a latent rule in each industry. If users often use mobile navigation, their personal life trajectory and whereabouts will leave traces in the system background, which makes users' private informa- 
tion leak out more easily. There are also some hidden dangers in the abuse of permissions of mobile APPs. When users book tickets, a Didi taxi, a catering takeout or go shopping or use navigation online, etc., they have to install phone APPs, while almost all the APPs ask for users to agree with them to have access to users' current position, contact lists, mobile phone number and identification number. What's more, these APPs also ask for users to enable them to use the mobile phone cameras, record messages and make a call. If users do not agree, they cannot install the software. So check it out and disable as many permissions as possible when an APP is installed. In addition, all photos taken by smart phones contain Exif parameters. When users take photos, time and place information will be accurately recorded due to the use of global positioning system. When users share the moments on WeChat, their personal information will be publicly exposed. Sometimes this will lead to a criminal crime. Now more and more criminal cases caused by sharing the moments are exposed.

\section{Government Measures against Public Opinion Monitoring in the Big Data Era}

Professor Wu Hequan, an academician in China, once said, "China, with the largest population in the world, will produce the most data. However, we do not pay enough attention to data storage and the utilization rate of stored data is not high. In addition, some departments and institutions in China have a large amount of data but are unwilling to share it with other departments, resulting in incomplete information or overlapping investment." [17] An important way to promote the construction of smart government is to establish professional public opinion institutions. By building a government public opinion data platform, opening up government data, attaching great importance to technology, talent training practices and service orientation, the government can reconstruct a public opinion management system to greatly improve its governance capacity.

\subsection{Build an Online Public Opinion Platform for Government and Improve Its Information Collection System}

The value of data is based on the data application, and improving the information collection is the first step to public opinion detection, analysis and warning. The construction of an efficient, all-round and multi-level data foundation platform is an important medium and guarantee for the government to improve the efficiency of data application. By virtue of its technical advantages, big data platform can collect massive data at a high speed for rinsing and screening to integrate high-value information. The use of cloud computing makes it easier to explore the value of big data. This computing mode of sharing resources through virtualization is the latest trend of information technology development after PC and Internet. How to take full advantage of cloud processing technology? The construction of a cloud platform can be realized in three steps [18]: data cloud construction, public opinion cloud construction and intelligence cloud construction. Data cloud is the central database established for all information, 
which has two powerful functions: data-searching and data-integrating. There are two kinds of information collection: whole network collection and directed collection. Public opinion cloud is the collection and integration of application software on the basis of data cloud, extracting the development rules of events, analyzing the development trend of public opinion and giving early warning of crisis events. Intelligence cloud integrates artificial intelligence technology with online politics on the basis of public opinion cloud and data cloud. Intelligence cloud is more intelligent and humanized than general public opinion monitoring software system, which can condense social governance policies, and has much more value in public opinion guidance.

\subsection{Open Data and Enhance the Government's Public Credibility}

The government, enterprises and public institutions have accumulated a large amount of data. In terms of management, they are the monopoly of some authoritative information, which leads to the fragmentation and poor communication of online public opinion data information among the organizations. To strengthen the information coordination among departments, it is necessary to break the "isolated information island" and "data dictatorship" status quo of public opinion so as to realize information sharing among major organizations, enterprises and public institutions, and maximize the development and creation of data value. The "big" of big data not only comes from a wide range of sources, but also is full of a lot of "useless and false information" with low value density and uneven data quality, which requires us to screen out the dross and select the essence. In addition, irrational factors also reduce the reliability of big data. This requires us to collect data and information as accurately, comprehensively and timely as possible, and try to maintain a rational attitude at the same time. If the research data is incomplete and unreasonable, the public opinion analysis report is likely to be misled, which will make it difficult for the government to grasp the overall situation of the crisis. It is likely for the government to make wrong decisions and let the government face a new round of public crisis.

All in all, the more data collected, the more accurate the prediction will be. Therefore, the sum of all the data is more valuable than the local data sampling. Big data processed in the era of online public opinion should be complete, namely, the data sample presented and analyzed should be overall, which urges the government to break the information barrier between different departments to show its diversification and high velocity in the network platform, to break through policymakers' closed unicity and isolated management ideas to intensify resource sharing among different departments, to bridge the information systems between big information institutions, and calls for government departments to constitute an open online data platform. Government Web3.0 is a government reform in order to adapt to the general trend of open data. It is individual-centered and takes the initiative in data opening and pays more attention to communication and cooperation so as to realize the sharing and co-governance between government and society. As Viktor puts forward: "When we combine 
the sum of multiple data sets, the value of the recombined sum itself is greater than that of the sum of the individual data sets." [19] For this reason, the $13^{\text {th }}$ Five-Year National Informatization Planning has clearly put forward: A unified and open national data resource system should be established. In 2018, a system of laws, regulations and policies on the open sharing of public data resources was established. At the same time, a unified platform for sharing, exchanging and opening national government data has been set up, and a shared pattern of data sharing across departments has basically taken shape. By 2020, government data sets in areas such as livelihood assurance services will be open to the public [10].

\subsection{Make Constant Efforts to Promote the Capacity of Research and Development, Technology Innovation and Professional Cultivation}

At present, online public opinion monitoring and analysis technology mainly relies on content analysis method and Web data mining. An automatic public opinion monitoring and management system based on the information from public opinion analysis engine thus takes shape to track topics, predict trends, prompt alarms and generate reports or visually present the characteristics and development trend of public opinion in the form of texts, graphics and other forms on the basis of comprehensive data mining and semantic analysis. The information from the automatic monitoring and management system includes online public opinion brief reports, special reports and early warning reports. However, the development speed of the Internet is much faster than that of technology. Especially since WeChat, microblog and other we-media became important platforms for netizens to express their opinions, the fragmentation and diversification of communication contents as well as the complexity of communication paths and modes have created new challenges and requirements for public opinion monitoring technologies and tools. Science and technology and talents are the two wings of a bird, which can maintain the power of online ideological discourse right. In addition, not until we occupy the commanding heights in the innovation of network technology and network talent training, will we really seize the initial opportunity to maintain our online ideological rights of speech [20]. Therefore, we should follow this trend, plan for and respond to the trend, firmly hold the "cow's nose" of core technology, grasp the network frontier breakthrough technology and the key internationally competitive core technology so as to make better use of data resources. At the same time, paying attention to the research and development and innovation of network public opinion data security and personal privacy protection technology, and using new technology to guarantee the public security of data and the right of civil free expression is the way of good governance in the era of big data. Xi Jinping said in his speech at the Symposium on Cybersecurity and Informatization, "In order to take the initiative in China's Internet development and ensure Internet security and national security, we must break through the core technology and try to 
overtake others in some fields and in some aspects." [21] All this requires the country and the government to make constant efforts to promote the capacity of research and development, technology innovation and professional cultivation.

\subsection{Improve Government Officials' Media Literacy and Strengthen the Awareness of Online Public Relations}

Government officials' media literacy, also known as leadership media literacy, includes the ability to recognize, understand, debate and use media, which is directly related to maintaining government image and good social relations. Government officials' media literacy demands a comprehensive understanding of mass media and takes a positive attitude and initiative to publicize the policy, shape the image, guide the masses, mold the authority, and disseminate scientifically and effectively on the basis of the understanding of the relationship between mass media and politics, economy, society and culture. Therefore, leadership quality is a kind of ability, which should be included in the compulsory courses for the training of party and government leading cadres at all levels. Meanwhile, the awareness of online public relations in the government decision-making, policy implementation and supervision should also be strengthened. In reference to a famous public relation expert Michael Regester's principle in his Crisis Management, the government work should, with full of the milk of human kindness information instead of the attitude of the business, make good use of interpersonal communication strategies to transmit the first time information truly and transparently in order to set up a good image of service-oriented government. The effective ways are as follows: Open government affairs microblog to inquire politics through network, deeply open the communication between the government and the public, ease social conflicts and control negative public opinions; Improve the early-warning mechanism of online public opinion crisis and respond quickly, accurately and ruthfully; Make good use of new media strategy, set an online agenda, and cultivate the mainstream media to become the core force of public opinion guidance; Strengthen the construction and study of Internet-related management laws and regulations, and improve the application cognition; Make full use of public opinion technology in government governance, government service and government innovation, timely grasp the development trend of public opinion, and finally achieve the expected effect of network media communication and public relations [22].

\subsection{Build Various Types of Case Data Analysis Database to Improve the Government's Mechanism}

At present, the Chinese government's research on public opinion focuses on the public opinion report issued in the process of emergencies and after the event and the awareness of online crisis public relations is not strong enough. There is a lack of regular interpretation of normalized events and the active correlation, evolution, prediction and analysis between normal events and public opinion events are rarely seen. Systematic studies on the early-warning mechanism, 
modeling and risk assessment of public opinion events need to be further improved. The construction of various types of online public opinion case database can provide reference for government decision-making by analyzing similar cases. The establishment of case data analysis database not only stores precious historical data for social development, but also facilitates the update of data analysis methods, so as to improve the government's ability to deal with events.

\section{Conclusion}

In short, we should fully understand the social value of big data, strengthen resource management and actively face with the urgent problems in the development of the Internet and urge the government to face with a new round of transformation in governance, which at the same time have brought new opportunities and challenges to social development and public opinion research. In addition, we should also construct a government platform for online public opinion to improve its information collection; employ an open data policy to improve public credibility in government; make full use of data value obtained from data mining and analysis technology, cultivate more professionals and maintain a sustainable development in research and development as well as technology innovation; improve government officials' media literacy and strengthen their awareness of online public relations; build various types of case data analysis database to improve the government function. All of these play an important role in realizing Intelligence China and remodeling government management system and capability and also are a prelude to the application and development of big data.

\section{Fund Program}

Philosophy and Social Science Project of Anhui Province 2015 (SN: AHSKY2015D157).

\section{Conflicts of Interest}

The author declares no conflicts of interest regarding the publication of this paper.

\section{References}

[1] Cai, L. and Yang, X. (2015) Research on the Application of Big Data in Social Public Opinion Monitoring and Decision-Making. Administrative Tribune, 2, 1.

[2] (2015) Forward-Looking Industrial Research Institute Release. Analysis Report on the Development Prospect and Investment Strategic Planning of China's Big Data Industry from 2015 to 2020 . http://blog.sina.com.cn/s/blog_c4a726e70102wkvp.html

[3] Sohu (2016) Big Data Expo: Ali Cloud Releases the World's First One-Stop Big Data Platform. http://www.sohu.com/a/55519430_267780

[4] Sohu (2019) Which Four Aspects Can the Characteristics of Big Data Be Summarized into? http://www.sohu.com/a/298387881_100116031

[5] Entertainment Prophet (2019) The First 5G Cell Phone Call Has Been Successful! 
How Long Will Your City Be Waiting for It? http://www.yingjunfalan.com/5724363/20190401A0DKA200.html

[6] Su, X. (2016) The 13th Five-Year Plan for National Economic and Social Development of the People's Republic of China.

http://www.china.com.cn/lianghui/news/2016-03/17/content_38053101.htm

[7] People's Daily Online-People's Daily Overseas Edition (2017) Xi Jinping Presided over the Collective Learning of the Political Bureau of the CPC Central Committee and Stressed the Promotion and Implementation of the National Big Data Strategy. http://world.people.com.cn/n1/2017/1211/c1002-29697998.html

[8] Dang, X. and Yuan, P. (2016) Difficulties and Solutions of Big Data Public Opinion Management. China Newspaper Industry, No. 5, 12.

[9] China Civilization Network (2018) The 42nd Statistical Report on the Development of Internet in China by CNNIC. http://www.wenming.cn/bwzx/dt/201808/t20180821_4801026.shtml

[10] Ministry of Industry and Information Technology of the People's Republic of China (2016) The 13th Five-Year National Informatization Plan (NDRC [2016] No. 73).

[11] Library Intelligence Work Publications (2014) Research and Dissemination of Online Public Opinions in the New Media Environment. China Ocean Press, 4, 3.

[12] People's Daily Online (2018) Scientific Decision-Making, Big Data as a First-Line Reference (Big Data Will Help National Governance). http://bbs1.people.com.cn/post/129/1/2/167326100.html

[13] New Horizon Online (2019) What Percentage Unstructured Data Now Accounts for the Total Human Data by Mr Tu Zi. http://power-bd.com/e-332325404535036285.html

[14] Tang, J. (2017) Ten Questions in New Media Communication. People's Daily Publishing House, 106.

[15] Xinhua (2018) Nanjing Think Tank Summit on Internet Big Data and Social Governance Was Held. http://www.xinhuanet.com/local/2018-01/27/c_129800411.htm

[16] Sohu (2018) Daily Top: Baidu's Mobile App. Monitors Users' Conversational Contents—The Truth Is Schoking. http://www.sohu.com/a/215117861_243181

[17] Wu, H. (2013) Seeking Truth: Opportunities and Challenges in the Era of Big Data. Communist Party of China News Network. http://theory.people.com.cn/n/2013/0216/c40531-20495578-3.html

[18] Lv, X. and Wang, H. (2014) Political Cloud Platform Construction Will Promote the Interaction between the Government and the Public and the Intensification of Information Processing-Based on the Research from Aoyi Network Platform and Huizhou Network Platform. E-Government, No. 12, 42.

[19] Mayer-Schönberger, V. (2013) Big Data: A Revolution That Will Transform How We Live, Work and Think. Zhejiang People's Publishing House, 39.

[20] Xie, X. (2018) The Political Wisdom of Maintaining the Right of Discourse in Network of Xi Jinping. Journal of Chongqing University of Technology (Social Science), No. 6, 143.

[21] Xi, J. (2019) On Internet Development: Core Technologies Are the Biggest "Lifeline”. Communist Party of China News Network. http://www.cac.gov.cn/2019-02/15/c_1124119850.htm

[22] Ao, Y. and Liu, T. (2017) The Influence of Micro Media on the Discourse Power of the Government Network and the Countermeasures. Journal of Chongqing University of Technology (Social Science), No. 4, 116. 